\title{
IOT Healthcare Communication System for IEEE 11073 PHD and IHE PCD-01 Integration Using COAP
}

\author{
Wei $\mathrm{Li}^{1}$, Cheolwoo Jung ${ }^{1}$ and Jongtae Park ${ }^{1}$ \\ ${ }^{1}$ School of Electronics Engineering, Kyungpook National University \\ Daegu, Korea \\ [liwei@ee.knu.ac.kr; cwjeong@ee.knu.ac.kr; jtpark@ee.knu.ac.kr] \\ *Corresponding author: Jongtae Park
}

Received August 7, 2017; revised October 30, 2017; accepted November 26, 2017; published April 30, 2018

\begin{abstract}
With the proliferation of the Internet of Things (IoT) healthcare devices, significant interoperability issue arises where devices use proprietary data transfer protocols. The IHE PCD-01 standard has been suggested for the exchange of healthcare data in ISO/IEEE 11073 PHD data model. However, the PCD-01 is not efficient to be used in the IoT environment. This is because the use of SOAP for PCD-01 may be too complex to be implemented in the resource-constrained IoT healthcare devices. In this paper, we have designed a communication system to implement ISO/IEEE 11073 and IHE PCD-01 integration using the IETF CoAP. More specifically, we have designed the architecture and procedures, using CoAP, to seamlessly transmit the bio-signal from the tiny resource-constrained IoT healthcare devices to the server in a standardized way. We have also built the agent, gateway, and PCD-01 interface at the server, all of which are using the CoAP as a communication protocol. In order to evaluate the performance of the proposed system, we have used the PCD data to be transmitted over CoAP, MQTT, and HTTP. The evaluation of the system performance shows that the use of CoAP results in faster transaction and lesser cost than other protocols, with less battery power consumption.
\end{abstract}

Keywords: IoT healthcare data communication standards, IHE PCD-01, ISO/IEEE 11073 PHD, CoAP, MQTT 


\section{Introduction}

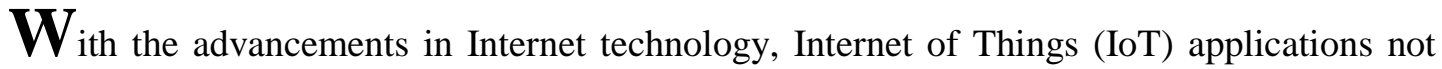
only bring convenience to our lives but also make our lives to have undergone tremendous changes. Meanwhile, the domains that use IoT have been diversified including healthcare, smart home, intelligent transportation, and smart cities. IoT means the various small embedded electronic devices collect and exchange data over the Internet [1]. More and more people realize the importance of health care and aspire to obtain medical services conveniently, which makes smart healthcare one of the important applicable fields of the IoT. Using various IoT devices, real-time healthcare monitoring and management of patients can be effectively achieved. However, many personal health devices are produced by various manufacturers who may use different communication protocols and standards, causing device interoperability problem, which is one of the main issues affecting smart healthcare.

In order to cope with the interoperability, compatibility and reliability issues, some research groups have developed a number of unified international standards. One of important standards is the ISO/IEEE 11073 personal health device (PHD), which enables an interoperable communication between diverse medical devices and healthcare servers. ISO/IEEE 11073 PHD standards family [2] consists of a "framework" standard and "device specialization" standards. As a framework standard, ISO/IEEE 11073 optimizes exchange protocol, defines domain information model, service model and the communication model to support device specialization [3]. The device specialization standards define the data model for the specific type of personal health device, such as pulse oximeter, blood pressure monitor, medication monitor, and so on. These specific medical devices encode the measurement parameters using ISO/IEEE 11073-20601 optimized exchange protocol, which may be transmitted through existing communication technologies such as Bluetooth and ZigBee [3].

ISO/IEEE 11073 PHD modeling includes domain information model (DIM) [4], service model and communication model. DIM specifies an object-oriented data model which can represent the structure and functions which are related to communication between medical devices. It defines a tree hierarchy for modeling the device and its behavior, which include measurements, alerts, or contextual data. ISO/IEEE 11073 PHD service model specifies the communication service such as get, set and event-report to access and control the information of the medical devices. ISO/IEEE 11073 PHD communication model manages the connection state and communication characteristics. ISO/IEEE 11073 family of standards may not be suitable for connecting and integrating tiny IoT devices to the hospital information system. In particular, the manager and agent architecture of the service model, and the mechanism of maintaining the communication connection state between agent and manager may be too complex to implement using the current architecture of the Internet of Things.

Various research attempts have been made to integrate the ISO/IEEE 11073 communication model with that of the Internet architecture. In [5], the authors propose a lightweight RESTful Web service approach, which uses 6LoWPAN to enable device management of wireless sensor devices. This approach supports a way to map the request/response message between CoAP and HTTP protocol. However, it does not consider the international health standards so that it cannot be directly used in the healthcare field. In [6], the authors propose a Constrained Application Protocol (CoAP) approach for PHDs communication, using IEEE 11073 as base health communication protocol. They propose 
how to carry IEEE 11073 information over CoAP messages. In [7], the authors present an IoT remote healthcare monitoring system that provides the patient`s conditions through the Web browser. In [8], the authors explore the use of the MQTT (Message Queue Telemetry Transport) lightweight protocol together with the ISO/IEEE 11073 standard for healthcare data monitoring. They present new ways of connecting PHDs in home networks to the Internet by the use of MQTT brokers, which will reduce the amount of data traffic. Although they propose a method of data transport protocol for PHD communication, they do not consider the remote management for PHDs.

In order to realize global health data interoperability, Health Level Seven International (HL7) provides a framework to define the language, structure and data types so that it is possible for seamless exchange, integration, sharing and retrieval of electronic health information between different hospital information systems [9]. Integrating Healthcare Enterprise (IHE) has defined the transaction to exchange patient care device (PCD) data between healthcare application systems. The PCD framework specifies conventions to represent the information model for medical devices embodied in the IEEE 11073 DIM, observing the syntactic and semantic conventions of HL7 v.2.6. [10]. However, IHE PCD framework uses SOAP-based web services for communication mechanism of PCD transactions [11]. The SOAP-based web services of the PCD transaction may be too heavy for resource-constrained tiny IoT healthcare devices, so that they may cause high power consumption and low processing speed. In [12], the authors have studied the connection of standard medical devices which is based on ISO/IEEE 11073 using Bluetooth HDP (Health Device Profile). Although they consider the integration of the ISO/IEEE 11073 and HL7 standards, they did not consider data communication protocol.

For resource-constrained devices, the IoT standard basically supports two communication protocol: CoAP and MQTT. The IETF CoRE Working Group has designed a specialized web transfer protocol, called CoAP (Constrained Application Protocol), for constrained nodes which are interconnected in the IoT environment. CoAP is based on the REST model, so that users can observe the resource directory of smart devices in a simple and easy way [13]. MQTT is a publish/subscribe messaging protocol which is widely being used for lightweight machine to machine communications [14]. Since MQTT is based on a client-server model, the message from sensor client is delivered to the user through a broker server over reliable TCP connection. Recently, in [15], the authors describe an implementation of an end-to-end remote monitoring platform based on the IEEE 11073 standards for PHD. It advocates the adoption of data standards and nomenclature to support semantic interoperability between health and ambient assisted living in platforms.

In this paper, we have proposed an efficient architecture to integrate the IHE PCD transaction with the ISO/IEEE 11073 information model using CoAP. More specifically, we have designed the architecture and procedures, using CoAP, to seamlessly transmit the bio-signal from the tiny resource-constrained IoT healthcare devices to the server in a standardized way [16]. We have also built the agent, gateway, and PCD interface at the server, all of which are using the CoAP as a communication protocol. In this way, an integrated healthcare system can efficiently provide remote healthcare monitoring services in a unified and reliable way, and in addition to the low power consumption. In [10], the authors have defined to use HL7 V2.6 Observation Reporting based on SOAP using HTTP for PCD data communications. For our proposed system, we choose CoAP to implement PCD data communication. Since CoAP is based on the REST model and by default bound to UDP, it is better than HTTP for IoT healthcare field and PCD data communication. 
In order to verify the performance of the proposed system architecture, we have used Mobius platform [17] as a remote server. Mobius supports oneM2M standard which is one of IoT standard supported by more than 200 participating partners throughout the world [18]. We have also compared the performance of the system with that of using PCD data over HTTP, MQTT, and CoAP. We have evaluated the performance of our system communication processes such as message size, round trip time per PCD data and battery power consumption. In [19], the performance of IoT protocols has been comparatively analyzed. However, the authors have only analyzed the performance qualitatively. Continua [20] has defined to use RESTful exchanges to establish the communication channel but use MQTT to exchange healthcare messages. In contrast to these works, we have analyzed the performance comparison using PCD-01 healthcare data, quantitatively over CoAP, MQTT, and HTTP. The evaluation of the system performance shows that the use of CoAP results in the faster transaction and lesser cost than other protocols, with less battery power consumption.

The originality and salient features of the paper are summarized below.

(1) We propose an integration architecture of ISO/IEEE 11073 PHD data model with IHE PCD-01 system using the IETF CoAP.

(2) The salient feature of the proposed methodology is that the proposed system architecture with low communication cost can be obtained due to the simplicity of using CoAP. It is suitable for IoT healthcare applications and services which require high reliability with low communication cost.

(3) The performance has been evaluated by comparing CoAP with MQTT for the integration ISO/IEEE 11073 with IHE PCD-01 system.

The remainder of this paper is organized as follows. In Section 2, we introduce related works about the overview of international health standards and CoAP protocol. In Section 3, we describe the architecture to integrate the IHE PCD transaction with the ISO/IEEE 11073 information model using CoAP. Section 4 describes implementation environment and the results of the proposed system. In Section 5, we show some experimental results to verify our implemented system performance. Finally, we conclude on the research.

\section{Related Work}

\subsection{International Healthcare Standards}

\subsubsection{ISO/IEEE 11073 PHD Standards}

ISO/IEEE 11073 DIM defines an agent to represent a set of objects, all of which have their respective attributes to describe measurement or status data that are used to communicate with the manager [21]. Fig. 1 shows the DIM of the ISO/IEEE 11073 PHD standards. All of the personal health devices have a particular Medical Device System (MDS) object. MDS object is used to represent a unique agent and the status of the agent. The agent uses the internal attitudes of MDS to share its information with the manager. The MDS may contain objects belonging to classes, each of which has different functions, such as the metric class which act as a base class for the numeric class, the real-time sample array class and so on. A single measurement is represented by the numeric class which applies for the 32-bit or 16-bit 


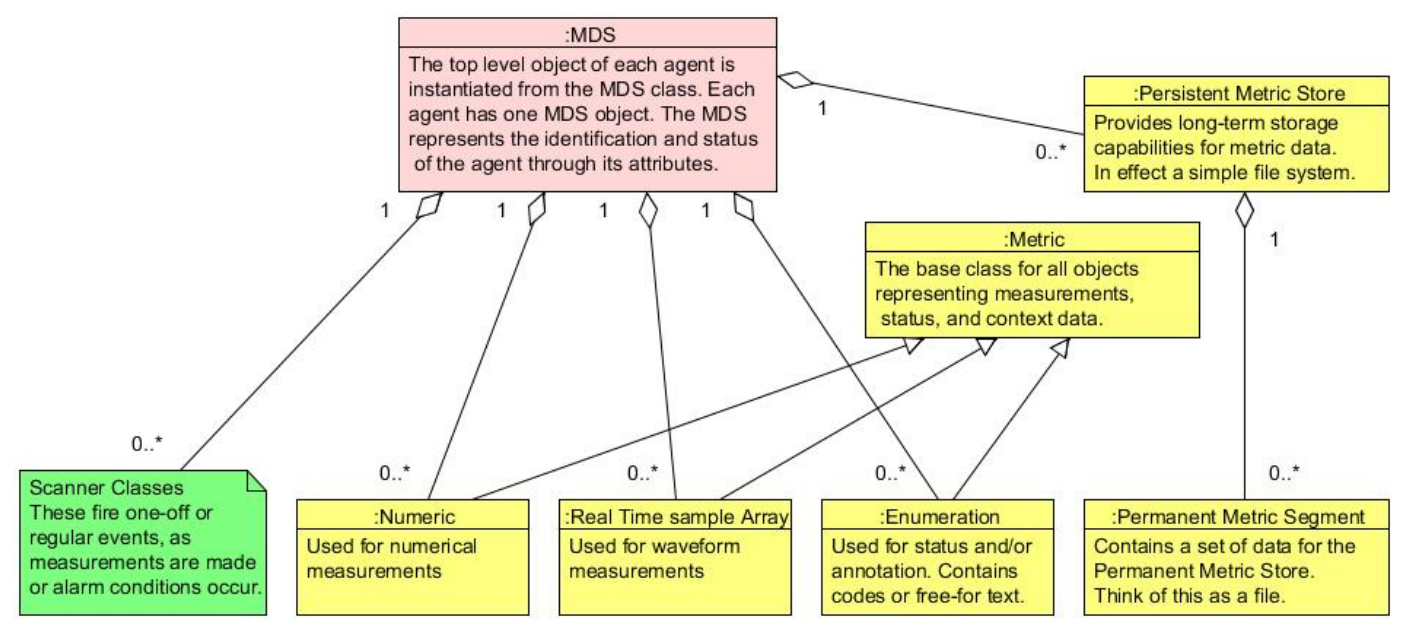

Fig. 1. ISO/IEEE 11073 PHD - DIM (Domain Information Model)

floating point number. And the continuous sample or waveform can be represented by the Real-Time Sample array class. In addition, the enumeration is used for status (codes) or annotations (text).

The service model defines the conceptual mechanisms to implement data exchange between the agent and the manager. The manager can access and control the domain information data of the agent.

The communication model supports the topology of point-to-point communication between multiple agents and a single manager. For each point-to-point connection, a connection state machine represents the dynamic system behavior (connection, association, and operation) by defining the states and sub-states of a pair of agent and manager. The communication model also defines in detail various communication operating conditions for each of the states including measurement data transmission. In order to send the data contained in an object, the communication model transforms the DIM data modeling based on ASN.1 into a "transfer syntax" and applies Medical Device Encoding Rules (MDER) to take the data and encode it into a binary message for communication. After receiving, the message can be explicitly encoded and parsed.

\subsubsection{HL7 (Health Level Seven) Standards}

HL7 standards are a set of international standards that focus on the application layer in the OSI model which include flexible standards, guidelines, and methodologies for transfer and management of clinical and regulatory data between healthcare systems used by various healthcare providers [9].

HL7 Standards are divided into three parts: Functional Standards, Semantic Standards, and Syntactic Standards. Syntactic Standards consist of Technical Standards (TCP/IP, encryption, security) and Exchange Standards (HL7 v.2 messaging, HL7 v.3 messaging and HL7 CDA, DICOM). Even Exchange Standards include Message Exchange Standards and Document Exchange Standards. It defines to specify the data exchange format, like HL7 v.2x, HL7 v.3, DICOM, NCPDP. 
HL7 v.2x messaging standard is the most recognized and widely implemented standard between various healthcare systems for clinical electronic data exchange in international healthcare domain. It is highly efficient and precise for clinical data management in central patient care systems [22].

The document exchange standards define the unified format of "Document" exchange. HL7 CDA is one electronic standard of the document exchange standards, it makes the exchange of clinical documents have a relatively high flexibility. Whether it is for people or machines, it has a very high readability because of its use of XML language. Any document that might have a signature (like a referral, clinical summary, history/physical examination, diagnostic report, prescription, or public health report.) is a viable document for CDA. CDA can be transported using HL7 V2, HL7 V3, DICOM, MIME-encoded attachments, etc. The high flexibility of CDA makes it be compatible with a wide range of environments and stored as a document in healthcare systems or transmitted as the content of a message [23].

\subsubsection{IHE PCD (Patient Care Device)}

Health information technology (HIT) systems and electronic health records (EHRs) can get a great interoperability by enhancing the use of standards designed by Integrating the Healthcare Enterprise (IHE) which belongs to an international propose. HIT proficient, IHE providers and other stakeholders in some clinical and operational areas can get a forum provided by IHE to achieve accordance with standards-based solutions to significant inter-operability problems. Use cases are related to the Patient Care Device (PCD) area where at least one actor like information system or a medical device exchange information with at least another actor defined as a patient-centric point of care clinical device. Semantic interoperability, real-time availability, and heterogeneity form the value of PCD profiles.

IHE PCD technical framework defines multiple integration modes for different integration requirements. Enterprise applications can get communication with multi-modality Patient Care Devices data and vendor independent provided by the device enterprise communication integration profile by using consecutive semantics, such as blood pressure, heart rate, etc. the Transmission of waveform data is represented by Waveform Content Module (WCM) integration schema, such as ECG waveform data, SpO2 waveform data, etc. Harmonizing the use of existing ISO/IEEE 11073-101011 nomenclature terms by systems compliant with IHE PCD profiles is the key purpose of the Rosetta Terminology Mapping

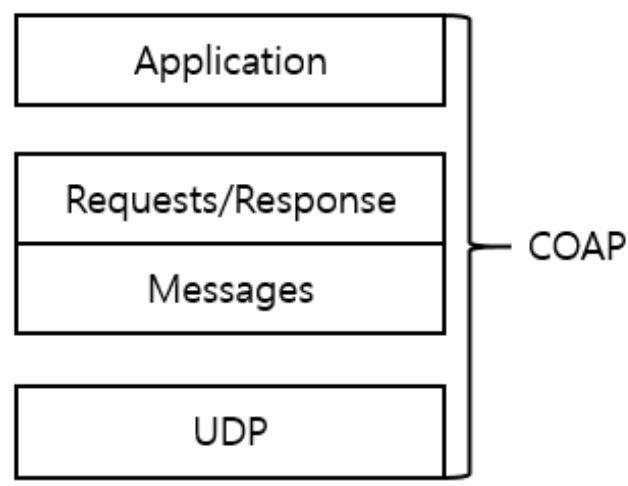

Fig. 2. Abstract levels of CoAP 
(RTM) managed value set. The units-of-measure and enumerated values allowed for every numeric element to help inter-operable and safe information exchanges among devices and systems are defined by the RTM profile. IHE-PCD profiles have to use RTM. Medical devices can be in accordance with their respective functions Asked, and choose to implement a number of integration patterns are defined in the role, can be satisfied Integration requirements [10].

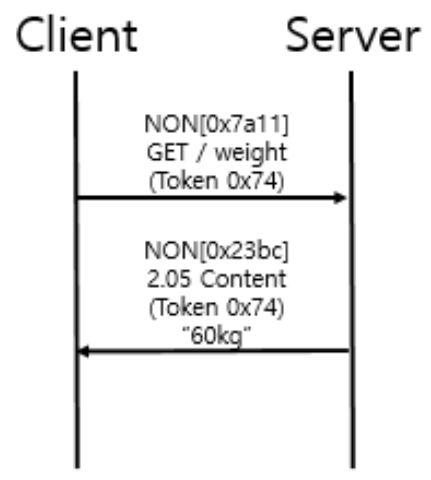

Fig. 4. Request/Response of NON message

\subsection{Constrained Application Protocol (CoAP)}

CoAP is designed as an application layer protocol for communication in tiny, low-power valves, switches, sensors and similar resource-constrained internet devices like WSN nodes. It is widely used in machine-to-machine (M2M) applications. The communication model of CoAP is similar to the client-server model in HTTP which exchanges messages through the request-response messaging pattern. Clients can access the resources generated by servers through GET, POST, Put, and DELETE methods. Unlike HTTP, CoAP is a datagram-oriented transfer protocol designed for asynchronous interaction and runs over UDP, not TCP hence ensures effective battery consumption through the connectionless model. In order to implement the process of request-response, CoAP defines four message types: CON, NON, ACK, and RST. Meanwhile, CoAP allows UDP broadcast and multicast to be used for addressing. The abstract levels are shown in Fig. 2.

The message model of CoAP is based on that of an end-to-end UDP communication. It is divided into two model types: CON (Confirmable) and NON (Non-Confirmable). The GET signaling flow of CON and NON are displayed in Fig. 3 and Fig. 4.

\section{Architecture of IoT Healthcare Communication System}

This section describes the architecture of IoT healthcare Communication System based on CoAP for ISO/IEEE 11073 and IHE PCD-01 integration and shows the details of its implementation. Fig. 5 shows its architecture, and the structure of the personal health device adaptor and personal health device observation reporter is shown in Fig. 6. 


\subsection{Architecture of IoT Healthcare Communication System}

\begin{tabular}{|c|c|c|c|c|c|c|}
\hline \multirow[b]{2}{*}{ Application Layer } & \multirow{2}{*}{$\begin{array}{c}\text { IEEE } 11073 \text { DIM } \\
\text { COAP }\end{array}$} & \multirow{2}{*}{\begin{tabular}{|c} 
IEEE 11073 DIM \\
COAP
\end{tabular}} & \multirow{2}{*}{$\begin{array}{c}\text { PCD-01 } \\
\text { CoAP }\end{array}$} & \multicolumn{2}{|c|}{ PCD-01 } & \multirow{2}{*}{$\begin{array}{l}\text { PCD-01 } \\
\text { HTTP }\end{array}$} \\
\hline & & & & COAP & HTTP & \\
\hline \multirow{2}{*}{ Transport Layer } & DTLS & DTLS & DTLS & DTLS & TLS & TLS \\
\hline & UDP & UDP & UDP & UDP & TCP & TCP \\
\hline Network Layer & IP & IP & IP & \multicolumn{2}{|c|}{ IP } & IP \\
\hline PHY/MAC Layer & LTE & LTE & Ethernet/WIFI & \multicolumn{2}{|c|}{ Ethernet/WIFI } & Ethernet/WIFI \\
\hline
\end{tabular}

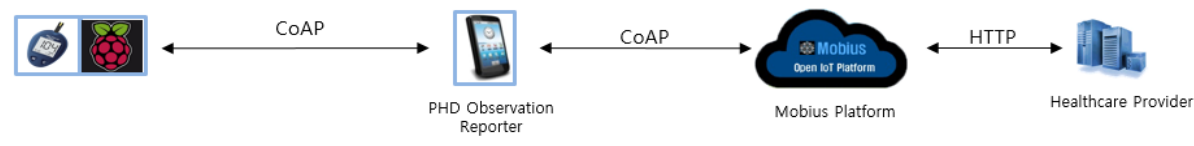

PHD Adaptor

8 Personal Health Device

Fig. 5. Architecture of the proposed communication system
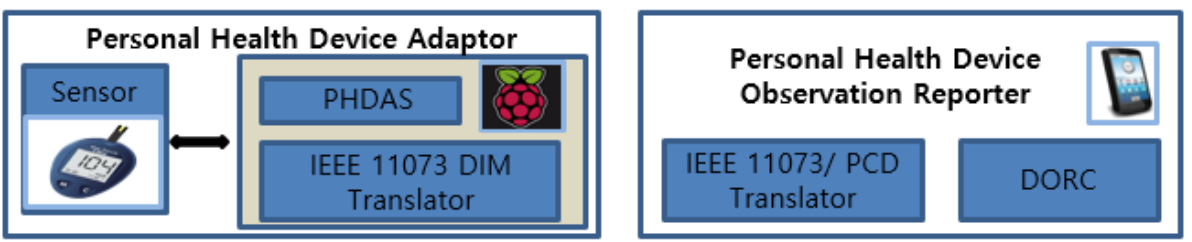

Fig. 6. PHD Adaptor and PHD observation reporter

\subsubsection{Personal Health Device Adaptor}

Personal Health Device Adaptor, which follows the ISO/IEEE 11073 PHD Standards, is defined to exchange data with personal health device observation reporter and for medical device data standardization to collect measured data from medical devices including standard devices and non-standard devices. This adaptor consists of PHDs Adaptor Software (PHDAS) and ISO/IEEE 11073 DIM Translator. PHDAS is defined to collect device data from the IoT device through Bluetooth or USB and connects with personal health device observation reporter. After registration, it sends the periodic or aperiodic measured data to the personal health device observation reporter using CoAP as the communication protocol. Moreover, it can revive remote control request for the connected device from the device observation customer through the Mobius platform.

ISO/IEEE 11073 DIM Translator is used to convert personal health device data into ISO/IEEE 11073 DIM data. As discussed previously, each personal health device is associated with the Medical Device System (MDS) object, which is defined by DIM. An MDS object includes Metric, PM-Store, PM-Segment, and Scanner. Although ISO/IEEE 11073 standards define MDER (Medical Device Encoding Rules) following ASN.1 (Abstract Syntax Notation One) language to describe device data, we select JSON (JavaScript Object Notation) to compile DIM in this paper. 


\subsubsection{Personal Health Device Observation Reporter}

Personal Health Device Observation Reporter is defined to receive device data and map the ISO/IEEE 11073 DIM data to PCD data, and then forward it to the Mobius platform and forward the control request from the Mobius platform to the personal health device adaptor for the remote control device. It consists of Device Observation Reporter Communicator (DORC) and ISO/IEEE 11073/PCD Translator. In order to achieve for interconnecting personal health devices, DORC is defined as a middleware platform. It includes the register, monitor and device management functions. In this paper, we implement the DORC platform in a smartphone application and use CoAP as the transport protocol instead of HTTP. In our designed application, it provides DORC function and personal information registration.

We have designed the ISO/IEEE 11073 DIM/PCD Translator to exchange data between ISO/IEEE 11073 DIM data and PCD data following the IHE PCD-01 framework.This translator can make communication data be converted between ISO/IEEE 11073 DIM data and PCD data. It follows the syntactic and semantic conventions of HL7 v2.x observation reporting to map ISO/IEEE 11073 DIM/Nomenclature to HL7 v2.x Observation Report and ISO/IEEE 11073 Data Types to HL7 Data types for the implementation of Transaction PCD-01. The IHE-PCD has identified the rules to map ISO/IEEE 11073-10101 DIM MDC Attributes to an HL7 ORU_R01 message as the PCD data used to communicate between DOR and DOC. After the DOC receives the message, it returns an HL7 acknowledgement to DOR. The ISO/IEEE 11073 DIM/PCD Translator analyses the ISO/IEEE 11073 DIM data from the personal health device to map it and the user Information that is created using the smartphone application to HL7 ORU_R01 data or map the ORU_R01 message which comes from the Mobius Platform to the ISO/IEEE 11073 DIM data.

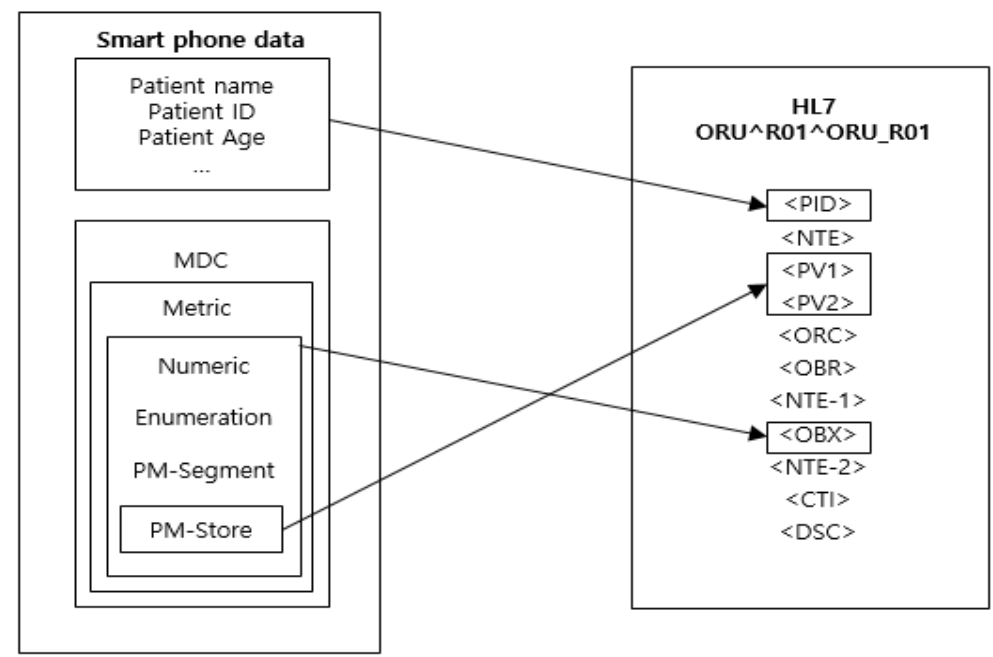

Fig. 7. Relation schema of ISO/IEEE 11073/PCD translator

The ORU^OR1^ORU_R01 message consists of some segments, including MSH (Message Header Segment), EVN (Trigger Event), PID (Patient Identification Segment), OBR (Observation Request Segment) or OBX (Observation Result Segment). The MSH 
segment defines the intent, source, destination, and some specifics of a message. The PID segment contains permanent patient identification, such as patient ID and demographic information, such as patient name. The relevant medical order information and observation properties are represented using OBR as the observation report header. OBX is an essential segment in ORU_R01 because it provides information on the device(s) from which measurements are generated or provides the related DIM information. However, all of these segments are mandatory except EVN. In order to implement the data mapping between the hierarchical structure of ISO/IEEE 11073 containment tree and a series of OBX messages, the ORU^OR1^ORU_R01 message structure provides a unified mechanism. If there are observation requests in the OBR field, the measured data of the Personal Health Device will be added to the OBX field to be sent. Fig. 7 shows the relational schema of the ISO/IEEE 11073/PCD translator. The translator implements the MDC code mapping, which is defined by the IHE PCD standards. For example, the reference ID of SpO2 is MDC_PULS_OXIM_SAT_O2, its code is 19384 and its partition number is 2. We get the MDC code which is used in the PCD data, using the following expression:

$$
\text { 2*65536+19384 = 150456(Partition code*2^16 +Term code) }
$$

\subsubsection{Personal Health Device Observation Consumer}

The personal health device observation customer is defined for remote monitoring and generating of EHR (Electronic Health Record), such as doctors in the hospital and private healthcare providers. They can obtain the special patient's past health information and remote real-time monitoring through the Mobius AE API based application after they are permitted by the patient.

\subsection{Signaling Flow of System}

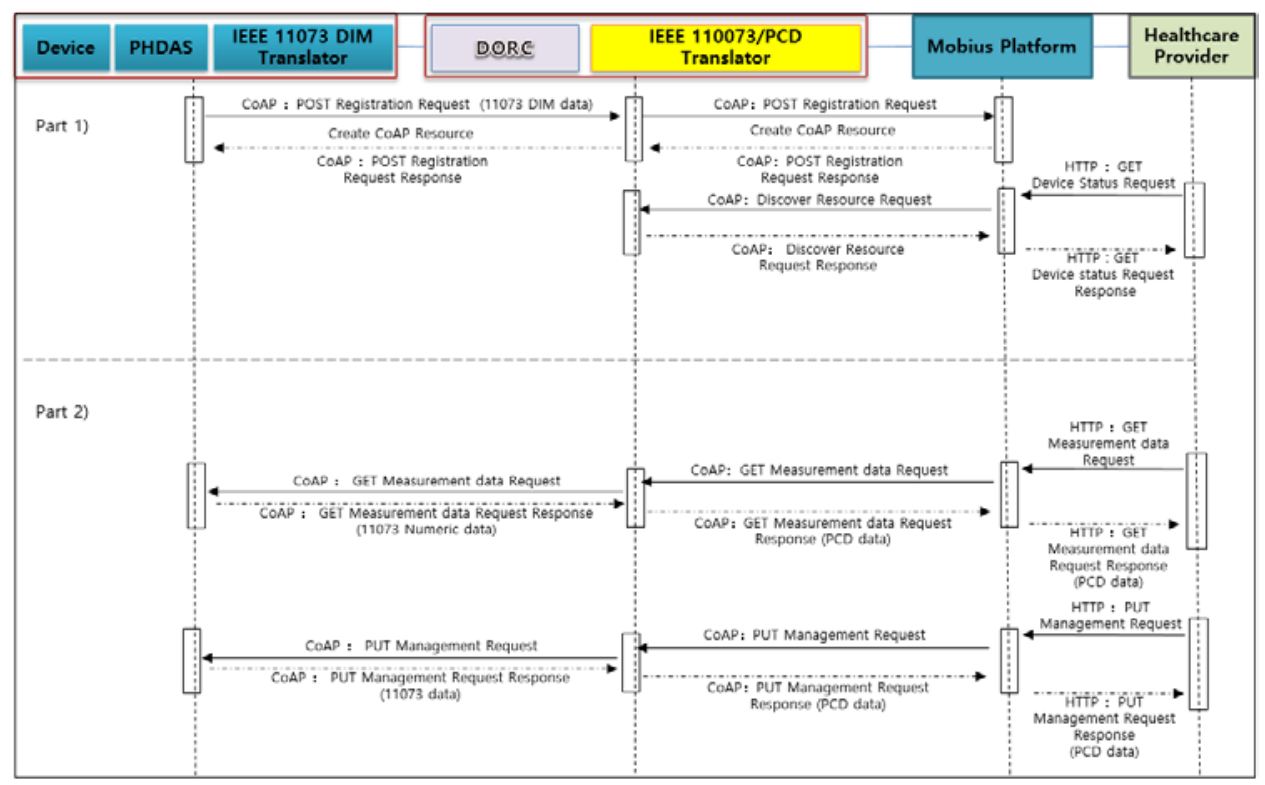

Fig. 8. Signaling flow diagram of system 
This section shows some signaling flow diagrams for the whole system in detail as shown in Fig. 8. We divide the signaling flow into two parts. Part 1 is the registration of personal health devices with the Mobius platform to support IoT services. Part 2 is the remote monitoring and management of personal health devices and healthcare providers.

\subsubsection{Healthcare Provider Remote Control}

For the remote control service, the Mobius platform applies the publish/subscribe communication model, which is defined by the MQTT. In this paper, we proposed a remote control model using CoAP for PCD-01, even pub/sub model of PCD-02 framework. First, the PHD adaptor discovers the PHD observation reporter resources. Then, the PHD adaptor uses CoAP observe method to register its interest in the MgmtCmd resource to the PHD observation reporter's CoAP server. Moreover, the PHD observation reporter discovers the Mobius platform resources and subscribes to the MgmtCmd resource in the Mobius platform. After completing the subscription of the device domain and PHD observation reporter to the MgmtCmd, the corresponding server adds the client to its list of observers of the MgmtCmd resource. Second, the healthcare provider or private manager uses the PUT method of CoAP to change the MgmtCmd resource state for remote control. Third, the Mobius platform sends a notification to the PHD observation reporter in the list of observers. Fourth, the PHD observation reporter maps the PCD data to ISO/IEEE 11073 DIM data from the Mobius platform and places the local CoAP server for changing the state of the MgmtCmd resource. Finally, the PHD observation reporter sends a notification to the device domain in the list of observers. Then, the device domain sets the device following the received ISO/IEEE 11073 DIM data. The The signaling flow is shown in Fig. 9.

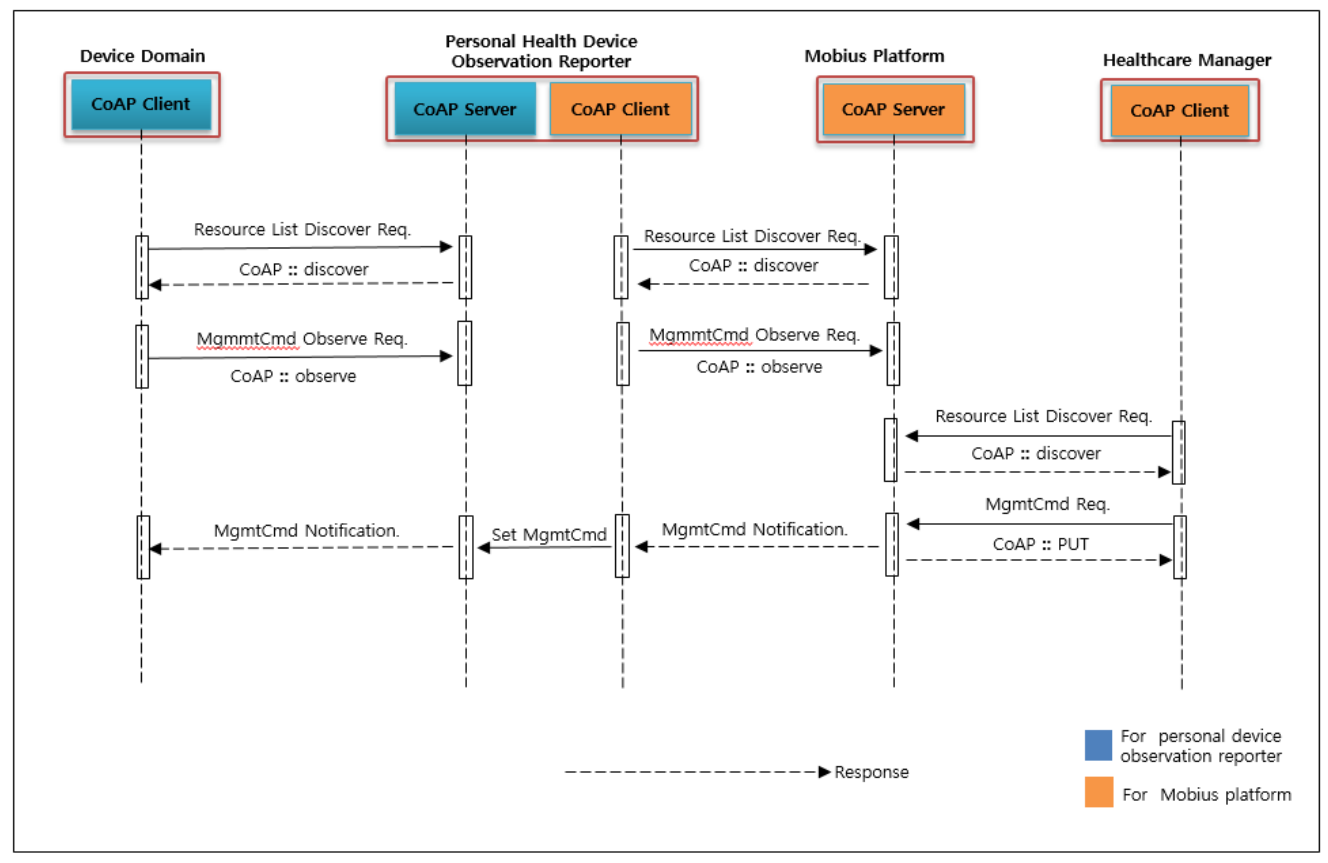

Fig. 9. Management signalling flow of system 


\subsubsection{Spontaneous Device Uploading of Measured Data}

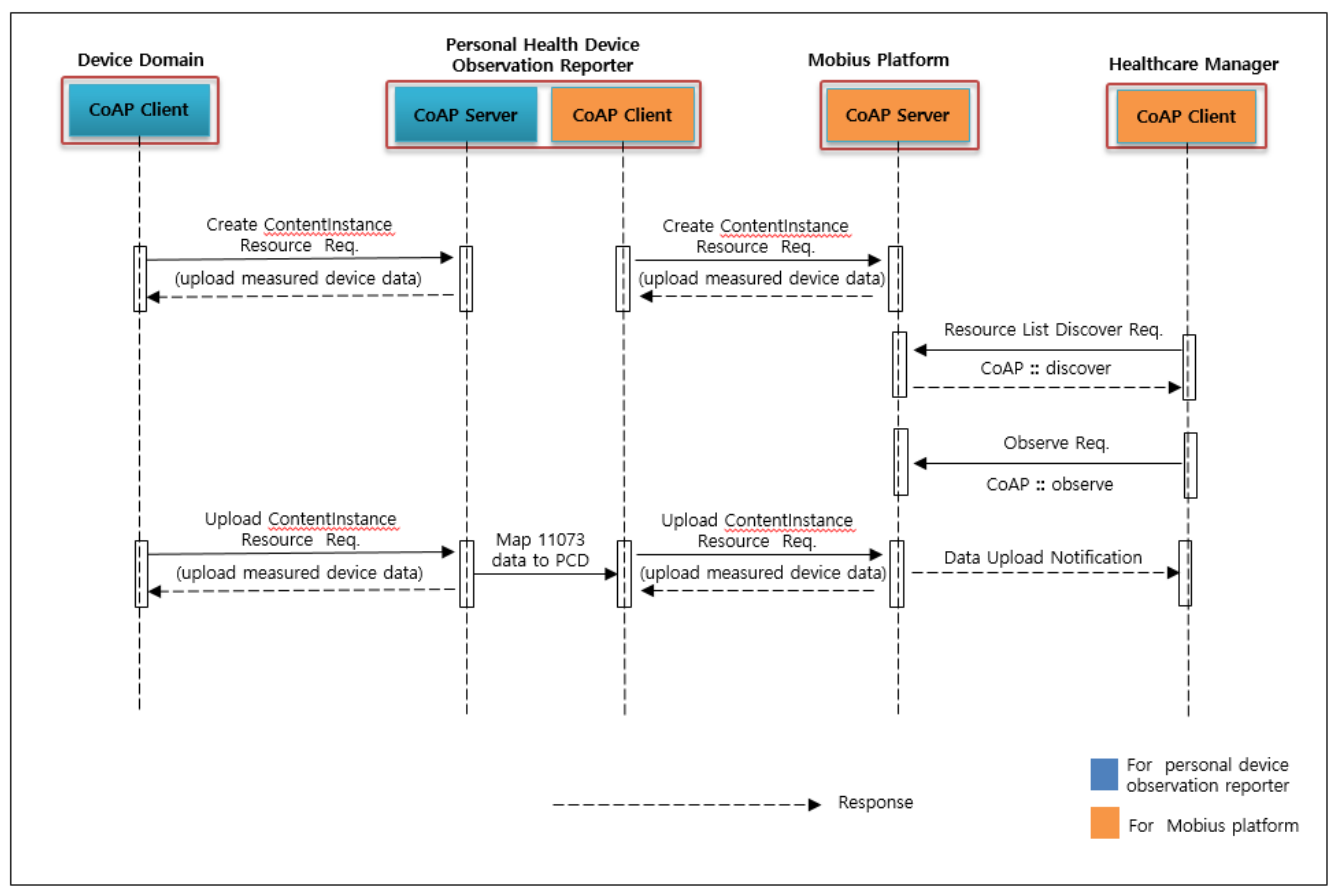

Fig. 10. Signaling flow diagram of device upload measured data

Fig. 10 shows that the device creates a contentInstance resource to the PHD observation reporter, and the PHD observation reporter creates the corresponding contentInstance resource to the Mobius platform for the device observation consumer to obtain the measured data. Then, the device sends the measured data to the PHD observation reporter spontaneously. After the PHD observation reporter receives the measured data, it maps ISO/IEEE 11073 DIM data to the PCD data and sends it to the Mobius platform for storage. We can obtain the PCD data, which is stored in the Mobius platform anytime. We expand the CoAP observe method to design this remote monitoring model following the PCD-01 transaction framework.

\section{Implementation of IoT Healthcare Communication System}

\subsection{Implementation Environment}

Table 1 shows the implementation environment of this communication system. We implemented our proposed IoT healthcare communication system for ISO/IEEE 11073/PCD-01 Integration Using CoAP as shown in Fig. 11. We show the process of the data measured by the Spo2 sensor generates the message following the IEEE 11073-10404 standard and its transmission. Moreover, we design a PHD Observation reporter application to collect and share the measurement data to the healthcare provider. Meanwhile, the reporter exchanges the data between the personal health devices and healthcare providers following the IEEE 11073 and PCD-01 standards. Then, we developed a health data monitor 
application, which can analyze the HL7 v2x-based PCD data to create an Electronic Health record.

Table 1. Implementation Environment

\begin{tabular}{|c|c|}
\hline Component & Environment \\
\hline Raspberry Pi & Raspbeny Pi 2, Spo2 sensor(HBE- spo2), Californium CoAP lib \\
\hline Computer & Intel i7, 10 GB ram, 64bit Windows 10 \\
\hline Smart Phone & Nexus 5[LGD821 model] (OS version 5.1) \\
\hline Development language & Java, Python \\
\hline
\end{tabular}

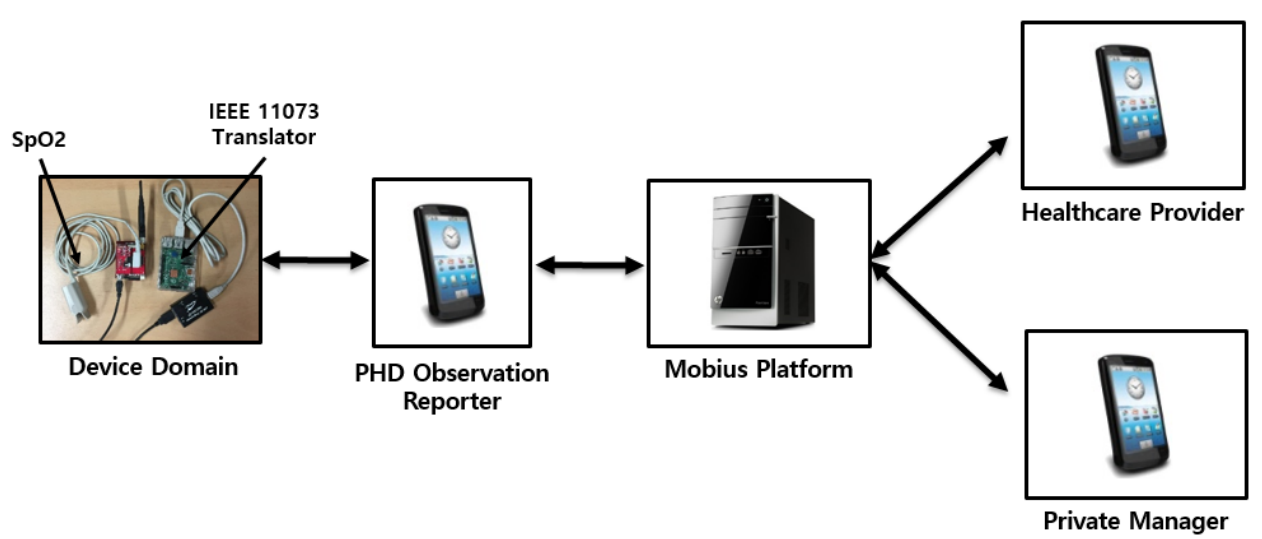

Fig. 11. Prototype of proposed system

In the experiment, we select a raspberry pi, a spo2 sensor, two Android smartphones and one computer. The raspberry pi acts as Personal Health Device Adaptor. The desktop computer acts as the Cloud to run the Mobius Platform. The Android smartphones act as the healthcare manager. We select the open source Antidote of Signove [24] to implement the ISO/IEEE 11073 DIM and CoAP server using California coap lib.

\subsection{Implementation Result}

Fig. 12 shows a sample of the PCD data generated by the ISO/IEEE 11073/PCD Translator. It shows the CoAP resource directory of the corresponding CoAP server. There are two registered users in $\mathrm{SpO} 2$. If the managers use the CoAP discover method, they can access the CoAP resource directory that belongs to a specific user ID. Then, healthcare providers can obtain the measured data by the health device that is registered by the user ID through the Mobius Platform. 


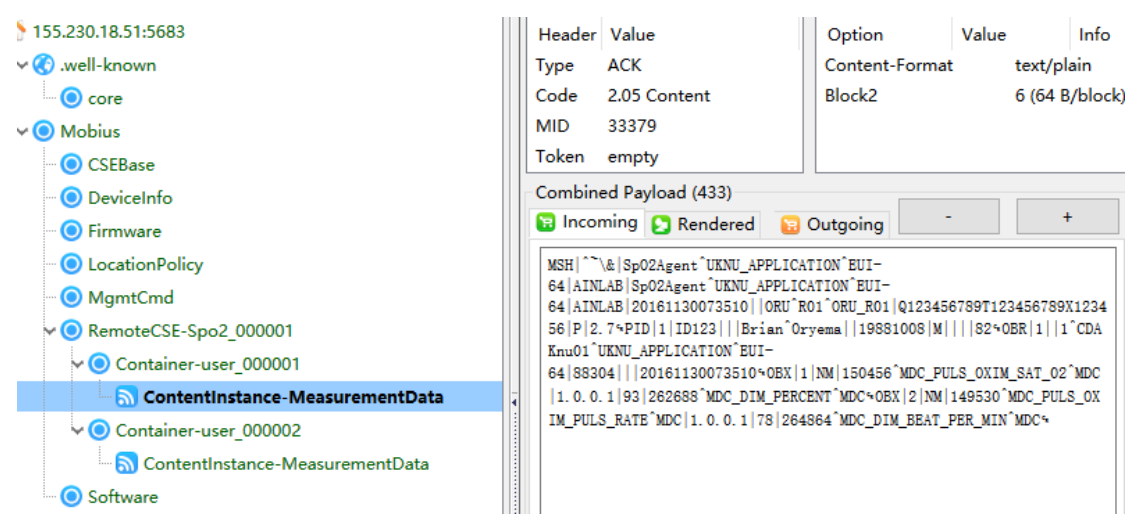

Fig. 12. Measurement PCD data on Mobius platform CoAP server

Fig. 13 shows the healthcare provider application. After the healthcare provider confirms a specific personal health device status, the provider can observe the measured data from that personal health device following the signaling flow, which is described in Section 3.
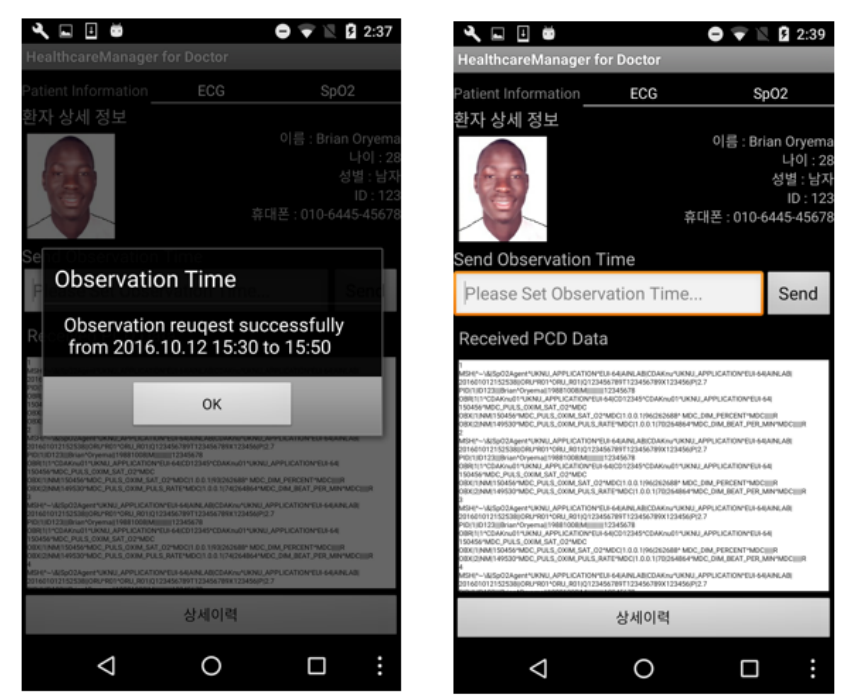

Fig. 13. Healthcare provider application

\section{Performance Evaluation}

In order to meet the requirement of IoT healthcare resource-constrained devices, we choose CoAP to transmit PCD data for our designed system. Therefore, we have evaluated the performance of our system communication processes such as message size, round trip time per PCD data and battery consumption. 


\subsection{PCD data over HTTP and CoAP Comparison}

Fig. 14 shows the round trip time per PCD data of CoAP and HTTP in the whole system. As we known, the communication speed of CoAP is faster than HTTP when they sent the same PCD data to the Mobius platform because the use of PCD data over CoAP also avoids the original three-way handshake mechanism. Although CoAP NON request is faster than the CON request given that the NON request is not a "Confirmable" message. We select to use the CON request for reliable communication. To save resources in the network and both at the client and server, we allowed the CoAP server to apply a piggybacked response of the $\mathrm{CON}$ as much as possible, which is carried directly in the acknowledgement message of the request (which requires that the request was carried in a Confirmable message). The RTT(Round Trip Time) per PCD data over HTTP is about 10 times longer than CoAP . This means that the data acquisition nodes of the transceiver and server require the high CPU processing time using the HTTP protocol, which has led to higher power consumption. Since CoAP is based on the Restful model, the cacheability feature of REST also reduces the consumption. We have also measured the battery consumption and compared it with the battery consumption of original PCD transmission based on SOAP over HTTP. The testing result is shown in Table 2. As shown in Table 2, the CoAP consumes less energy, fitting the resource-constrained devices.

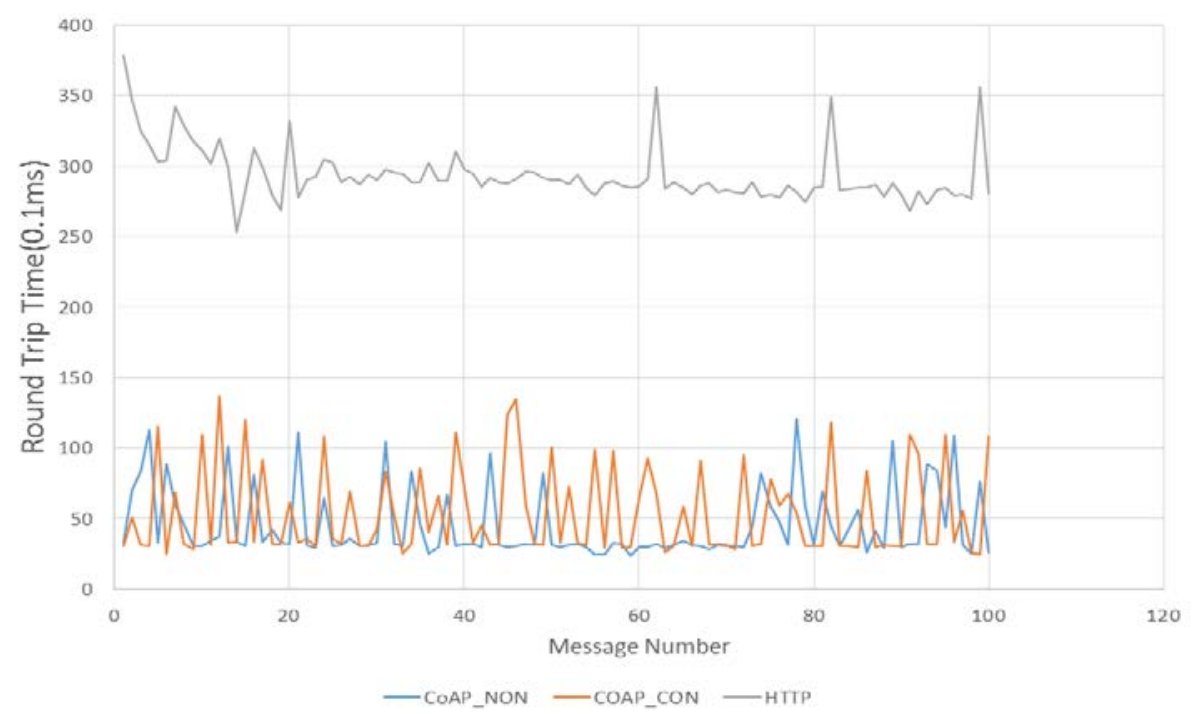

Fig. 14. RTT per PCD data for CoAP and HTTP

Table 2. Average Battery Consumption of PCD data over HTTP and CoAP

\begin{tabular}{|c|c|c|}
\hline & PCD data over HTTP & PCD data over CoAP \\
\hline Battery/Hour (Send) & $3.26 \%$ & $1.35 \%$ \\
\hline Battery/Message (Send) & $0.00092 \%$ & $0.00001 \%$ \\
\hline Battery/Hour (Receive) & $5.33 \%$ & $2.12 \%$ \\
\hline Battery/Message (Receive) & $0.00112 \%$ & $0.00012 \%$ \\
\hline
\end{tabular}




\subsection{Remote Control Model Performance}

For remote control, [20] and Mobius platform use MQTT publish/subscribe theory to implement authenticated the persistent session. In this paper, we have designed the remote control model based on CoAP to implement the PCD-01 framework instead of pub/sub model which defined by MQTT. We compare the transaction packet condition and round-trip time of the remote control model for PCD data communication based on CoAP and MQTT. Fig. 15 shows that the remote control model based on CoAP is faster than MQTT for PCD data communication. This is because MQTT has a slower transmit cycle in comparison to CoAP. Fig. 16 shows remote control transaction packet size of PCD data over CoAP and MQTT. Table 3 shows the battery power consumption for PCD data communication over CoAP and MQTT. Given that the MQTT uses TCP and needs a three-way handshake, our proposed model uses fewer internet resources, resulting in the faster transaction and less cost than MQTT.

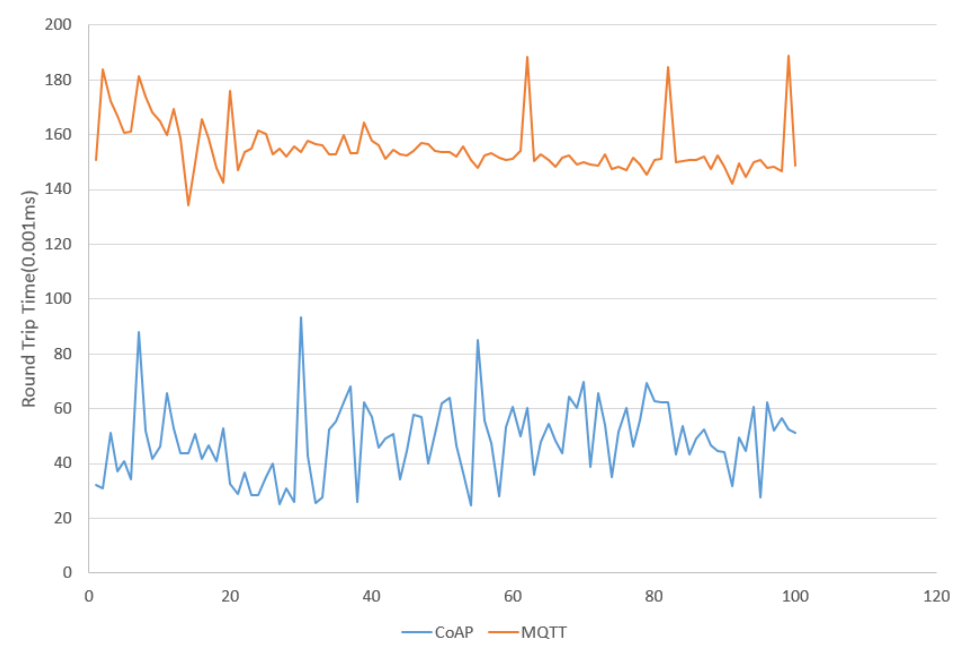

Fig. 15. Remote control model RTT per PCD data over CoAP and pub/sub of MQTT

Table 3. Average Battery Consumption of PCD data over MQTT and CoAP

\begin{tabular}{|c|c|c|}
\hline & PCD data over MQTT & PCD data over CoAP \\
\hline Battery/Hour (Send) & $4.15 \%$ & $1.35 \%$ \\
\hline Battery/Message (Send) & $0.00003 \%$ & $0.00001 \%$ \\
\hline Battery/Hour (Receive) & $3.54 \%$ & $2.12 \%$ \\
\hline Battery/Message (Receive) & $0.00015 \%$ & $0.00012 \%$ \\
\hline
\end{tabular}




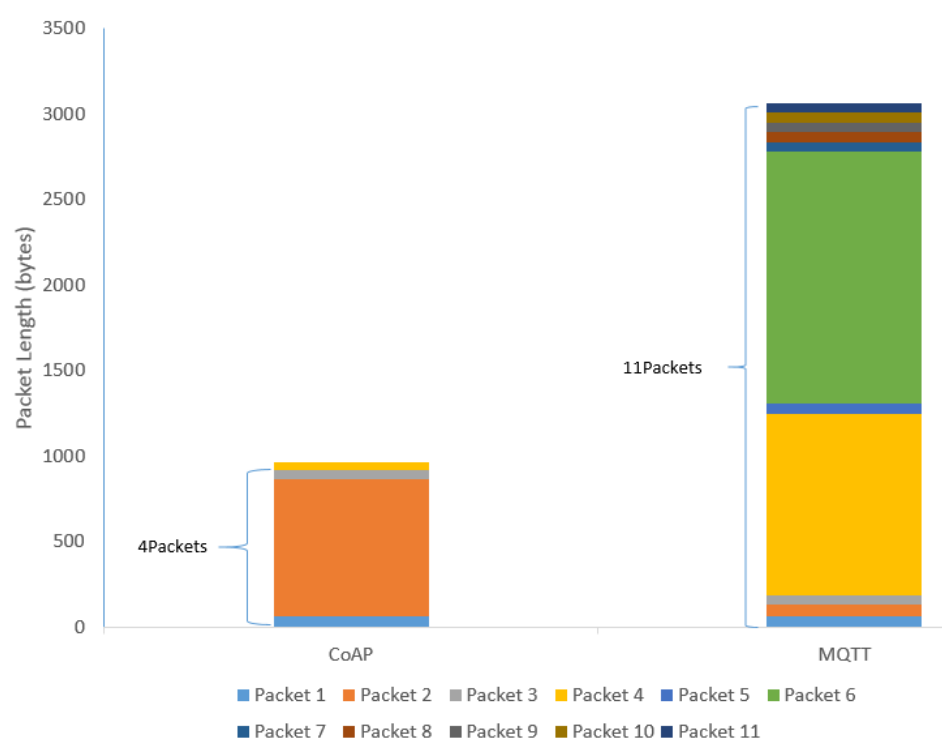

Fig. 16. Remote control model transaction packet length about PCD data over CoAP and MQTT

\section{Conclusion}

In this paper, we have designed and implemented a communication system using CoAP for the integration of ISO/IEEE 11073 and IHE PCD-01. We have built the ISO/IEEE 11073 and IHE PCD-01 integration gateway, and PCD-01 interface at Mobius server using the CoAP as a communication protocol. The proposed communication system allows the measurement data from the resource-constrained IoT healthcare devices to be reliably delivered to the server in a standardized way. We have also evaluated the performance of the proposed system like message size, RTT and battery consumption. The evaluation of the system performance shows that the use of CoAP consumes fewer Internet resources, resulting in the faster transaction than MQTT and HTTP for PCD data communication.

The ISO/IEEE 11073 and IHE PCD translator enables the healthcare provider to obtain the healthcare measurement data after verification and enhances interoperability between personal health devices and healthcare providers. The translator allows the personal healthcare data monitored at the various IoT devices to be seamlessly integrated with the electronic health record in the hospital information system.

\section{Acknowledgements}

This study was supported by the BK21 Plus project funded by the Ministry of Education, Korea (21A20131600011). This research was supported by Basic Science Research Program through the National Research Foundation of Korea (NRF) funded by the Ministry of Science and ICT(NRF-2016R1A2B4010551). This work was supported by Institute for Information \& communications Technology Promotion (IITP) grant funded by the Korea government(MSIP) (No.2016-0-00067,Wise-IoT). 


\section{References}

[1] U. Mulligan, and R. Brennan, "oneM2M: The Platform Standard for the Internet of Things," Sand Hill, January, 2015. Article (CrossRefLink)

[2] IEEE 11073 Personal Health Device. Retrieved on October 27, 2017. Article (CrossRefLink).

[3] "ISO/IEEE International Standard for Health Informatics Personal Health Device Communication Part 20601: Application Profile Optimized Exchange Protocol,” ISO/IEEE Std. 11073-20601, 2016. Article (CrossRefLink)

[4] "ISO/IEEE International Standard for Health Informatics Point-of-care medical device communication Part 10201: Domain information model,” ISO/IEEE Std. 11073-10201, 2004. Article (CrossRefLink)

[5] Z. Sheng, H. Wang, C. Yin, X. Hu, S. Yang, and C.M Leung, "Lightweight Management of Resource-Constrained Sensor Devices in Internet of Things," IEEE INTERNET OF THINGS JOURNAL, vol. 2, no. 5, October, 2015. Article (CrossRefLink)

[6] F.S. Santos, M. Bulitz, O. Almeida, and A. Perkusich, "Integrating IEEE 11073 and Constrained Application Protocol for Personal Health Devices," in Proc. of Symposium on Applied Computing, 2014. Article (CrossRefLink)

[7] D. Ugrenovic, and G. Gardasevic, "CoAP protocol for Web-based monitoring in IoT healthcare applications," in Proc. of 23rd Telecommunications Forum (TELFOR 2015), Belgrade, Serbia, pp. 24-26, November, 2015. Article (CrossRefLink)

[8] F. Gomes, F. S. Santos, O. Almeida, and A. Perkusich, "Integrating MQTT and ISO/IEEE 11073 for Health Information Sharing in the Internet of Things," in Proc. of IEEE International Conference on Consumer Electronics (ICCE), 2015. Article (CrossRefLink)

[9] Heath Level 7 Resource Library. Retrieved on October 27, 2017. Article (CrossRefLink)

[10] Patient Care Device (PCD) Technical Framework. Retrieved on October 27, 2017. Article (CrossRefLink)

[11] IHE IT Infrastructure Technical Framework Volume 1(ITI TF-1). Retrieved on October 27, 2017. Article (CrossRefLink)

[12] S. Oh, "A Study on HL7 Standard Message for Healthcare, System Based on ISO/IEEE 11073," International Journal of Smart Home, vol. 9, no. 6, pp. 113-118, 2015. Article (CrossRefLink)

[13] Z. Shelby, K. Hartke, and C. Bormann, "The Constrained Application Protocol (CoAP)," RFC 7252, June 2014. Article (CrossRefLink)

[14] MQTT Standard Version 3.1.1. Retrieved on October 27, 2017. Article (CrossRefLink)

[15] M. Clarke, J. de Folter, V. Verma, and H. Gokalp, "Interoperable End-to-End Remote Patient Monitoring Platform based on IEEE 11073 PHD and ZigBee Health Care Profile," IEEE Transactions on Biomedical Engineering, Issue 99, 2017. Article (CrossRefLink)

[16] L. Wei, "Design and Implementation of IoT Healthcare Communication System based on CoAP for IEEE 11073/PCD-01,” Master Thesis, School of Electronics Engineering, Kyungpook National University, South Korea, 2017.

[17] Open alliance for IoT standard (OCEAN). Retrieved on October 27, 2017. Article (CrossRefLink)

[18] Standards for M2M and the Internet of Things. Retrieved on October 27, 2017. Article (CrossRefLink)

[19] M. Anusha, E. S. Babu, L. S. Mahesh Reddy, A. V. Krishna, and B. Bhagyasree, "Performance analysis of data protocols of internet of things: a qualitative review," International Journal of Pure and Applied Mathematics, Vol. 115, no. 6, pp. 37-47, 2017. Article (CrossRefLink)

[20] Fundamentals of Data Exchange. Retrieved on October 27, 2017. Article (CrossRefLink)

[21] "ISO/IEEE Standard for Health Informatics Point-of-care medical device communication Part 10201: Domain information model,” ISO/IEEE Std. 11073-10201, 2014. Article (CrossRefLink)

[22] Heath Level 7 Version 2 Product Suite. Retrieved on October 27, 2017. Article (CrossRefLink)

[23] Heath Level 7 Clinical Document Architecture (HL7 - CDA). Retrieved on October 27, 2017. Article (CrossRefLink)

[24] Antidote: IEEE 11073 stack library. Retrieved on October 27, 2017. Article (CrossRefLink) 

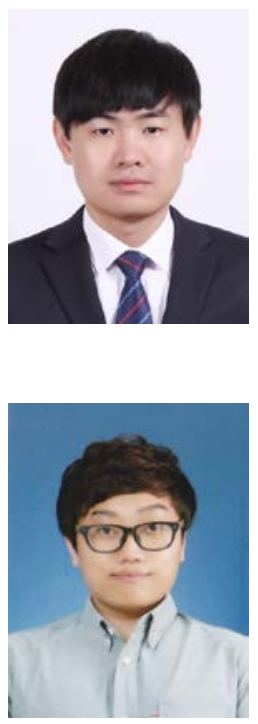

Wei Li received B.S degree from Electronic Engineering in Kyungpook National University, Daegu, Korea in 2015, and M.S degree in Kyungpook National University, Daegu, Korea in 2017. His research interest includes IoT healthcare service, communication and data exchange standardization.

Cheolwoo Jung received B.S degree from IT contents in Daegu Hanny University, Daegu, Korea in 2016, He is currently M.S in Electronic Engineering, Kyungpook National University, Daegu, Korea. His current research interests include IoT healthcare service and AI deep learning.

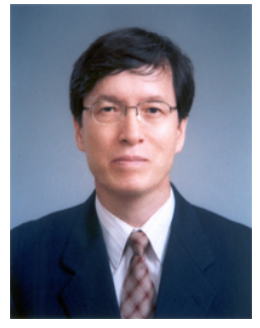

Jong-Tae Park, received the Ph.D. degree in Computer Science and Engineering from the University of Michigan. He is a professor in the School of Electronics Engineering, Kyungpook National University, Daegu, Korea. He previously worked at AT\&T Bell Labs in the United States. He has founded the Committee of Korean Network Operations and Management (KNOM) in the Korean Institute of Communication Sciences and was one of the founding members of Asia-Pacific Symposium on Network Operations and Management (APNOMS). He served as a chair of the Technical Committee of Information Infrastructure of IEEE Communication Society. He is currently on the editorial board China Communications. He was the general chair for APNOMS97, the general chair for ICC 2002 Symposium, and co-chair for Globecom2002 Symposium on Global Service Portability and Infrastructure. He has also served as a committee member or advisory board member for IEEE/IFIP NOMS and IM. He currently is a director of the center for U-healthcare Convergence Network. He has published more the 100 journals and articles in the areas of computer communication networks, network management, and distributed systems. His current research interests include the management of MPLS/GMPLS networks, fixed-mobile convergence technology U-Healthcare System, security and seamless mobility in next-generation heterogeneous broadband wireless networks, and ubiquitous sensor networks. He is a senior member of the IEEE. 